\title{
Preimages for the Tillich-Zémor Hash Function
}

\author{
Christophe Petit^ and Jean-Jacques Quisquater \\ UCL Crypto Group ${ }^{\star \star}$ \\ Universit catholique de Louvain \\ Place du levant 3 \\ 1348 Louvain-la-Neuve, Belgium \\ christophe.petit@uclouvain.be, jjq@uclouvain.be
}

\begin{abstract}
After 15 years of unsuccessful cryptanalysis attempts by the research community, Grassl et al. have recently broken the collision resistance property of the Tillich-Zémor hash function. In this paper, we extend their cryptanalytic work and consider the preimage resistance of the function.

We present two algorithms for computing preimages, each algorithm having its own advantages in terms of speed and preimage lengths. We produce theoretical and experimental evidence that both our algorithms are very efficient and succeed with a very large probability on the function parameters. Furthermore, for an important subset of these parameters, we provide a full proof that our second algorithm always succeeds in deterministic cubic time.

Our attacks definitely break the Tillich-Zémor hash function and show that it is not even one-way. Nevertheless, we point out that other hash functions based on a similar design may still be secure.
\end{abstract}

\section{Introduction}

The Tillich-Zémor hash function was one of the oldest unbroken cryptographic hash functions in the literature. It was proposed at CRYPTO'94, following the cryptanalysis of a related scheme of Zémor [15]16 14 12]. It received significant cryptanalytic attention over the years 3511110] but although closely related schemes had been completely broken [2139], it remained essentially intact during 15 years.

In August 2009, Grassl et al. introduced a new and very elegant algorithm finding collisions for the Tillich-Zémor hash function [6]. The authors discovered a particular structure in the hash values of palindromic messages (messages such that their bitstring representation can be reversed without changing) and exploited their finding with a nice result of Mesirov and Sweet [8] on the Euclidean algorithm applied to polynomials in characteristic 2.

In this paper, we extend the work of Grassl et al. to the problem of finding preimages for the Tillich-Zémor hash function. We first show that a tiny

\footnotetext{
* Research Fellow of the Belgian Fund for Scientific Research (F.R.S.-FNRS) at Universit catholique de Louvain (UCL).

** Supported by the Interuniversity Attraction Pole (IAP) projet BCrypt.
} 
modification of their algorithm actually provides a second preimage algorithm. Inspired by previous work on a similar hash function [9], we then reduce the problem of finding preimages to any hash value to the problem of precomputing preimages to a few hash values with certain characteristics. Finally, we provide two algorithms for this precomputing part.

Both our precomputing algorithms are very efficient and successful for random choices of the function parameters. Each algorithm has its own advantages resulting from different approaches. The first algorithm produces shorter preimages than the second one and it is therefore more interesting from a practical point of view. On the other hand, the second algorithm is deterministic and it is faster than the first one. It is also more interesting from a theoretical point of view since we have a proof that it always succeeds in deterministic cubic time for an important subset of the function parameters.

The remainder of this paper is organized as follows. In Section 2 we introduce our notations, the Tillich-Zémor hash function, the essential of Grassl et al.'s algorithm, and we briefly sketch out our algorithms. In Section 3, we modify Grassl et al.'s algorithm into a second preimage algorithm. In Section 4, we reduce the preimage problem to a precomputation part. In Sections [5] and 6] we give our two precomputation algorithms. We conclude the paper in Section 7 with a discussion of our results and the security of Tillich-Zémor-like hash functions. Finally, we illustrate our algorithms with a toy example in Appendix A

\section{Preliminaries}

\subsection{The Tillich-Zémor Hash Function}

Let $n$ be a positive integer and let $p(X)$ be an irreducible polynomial of degree $n$ over the field $\mathbb{F}_{2}$. Let $A_{0}$ and $A_{1}$ be the following two matrices

$$
A_{0}:=\left(\begin{array}{cc}
X & 1 \\
1 & 0
\end{array}\right) \text { and } A_{1}:=\left(\begin{array}{cc}
X & X+1 \\
1 & 1
\end{array}\right)
$$

that have determinant 1 . We call these matrices the generators of the TillichZémor hash function. Let $m=m_{1} m_{2} \ldots m_{k} \in\{0,1\}^{*}$ be the bitstring representation of a message. The Tillich-Zémor hash value of $m$ is defined as

$$
H\left(m_{1} m_{2} \ldots m_{k}\right):=A_{m_{1}} A_{m_{2}} \ldots A_{m_{k}} \bmod p(X) .
$$

\subsection{Notations}

Let $K:=\mathbb{F}_{2}[X] /(P(X)) \approx \mathbb{F}_{2^{n}}$. The images of the Tillich-Zémor hash functions are the matrices of the group $S L(2, K)$, that is the group of matrices with elements in $K$ and determinant 1. Let

$$
h\left(m_{1} \ldots m_{k}\right):=A_{m_{1}} A_{m_{2}} \ldots A_{m_{k}}
$$


be the Tillich-Zémor hash function without modular reduction. Its images are elements of $S L\left(2, \mathbb{F}_{2}[X]\right)$. In this paper, we sometimes identify the elements of $K$ to their unique representatives of degree smaller than $n$ in $\mathbb{F}_{2}[X]$. To remove any ambiguity when it may appear, we use the symbol = to mean an equality over $\mathbb{F}_{2}[X]$ and $\equiv$ to mean an equality over $K$. For $q(X) \in \mathbb{F}_{2}[X]$, we write $q_{i}$ for the coefficient of the term of degree $i$ of $q(X)$. Finally, if $m, m^{\prime} \in\{0,1\}^{*}$ are two bitstrings, we write $\mathrm{mm}^{\prime}$ for their concatenation.

\subsection{Grassl et al.'s Collision Algorithm}

Grassl et al. 6] first observed that two messages collide for the Tillich-Zémor hash function if and only if they collide for the following modified function

$$
H^{\prime}\left(m_{1} \ldots m_{k}\right):=A_{m_{1}}^{\prime} A_{m_{2}}^{\prime} \ldots A_{m_{k}}^{\prime} \bmod p(X)
$$

where

$$
A_{0}^{\prime}:=A_{0}=\left(\begin{array}{rr}
X & 1 \\
1 & 0
\end{array}\right) \text { and } A_{1}^{\prime}:=A_{0}^{-1} A_{1} A_{0}=\left(\begin{array}{rr}
X+1 & 1 \\
1 & 0
\end{array}\right) .
$$

Grassl et al. then observed the following property of palindromic messages. Let $h^{\prime}$ be the modified Tillich-Zémor hash function without reduction.

Proposition 1. 6] Let $m \in\{0,1\}^{2 k}$ be a palindrome of even length, say $m=$ $m_{k} \ldots m_{1} m_{1} \ldots m_{k}$. Let $a_{(0)}, \ldots, a_{(k)}$ be the following polynomials

$$
a_{(i)}= \begin{cases}1, & \text { if } i=0 \\ X+m_{1}+1, & \text { if } i=1 \\ \left(X+m_{i}\right) a_{(i-1)}+a_{(i-2)}, & \text { if } 1<i \leq k .\end{cases}
$$

Then $h^{\prime}(m)=\left(\begin{array}{cc}a^{2} & b \\ b & d^{2}\end{array}\right)$ for $a=a_{(k)}, d=a_{(k-1)}$ and some $b \in \mathbb{F}_{2}[X]$. Moreover, $h^{\prime}(0 m 0)+h^{\prime}(1 m 1)=\left(\begin{array}{cc}a^{2} & a^{2} \\ a^{2} & 0\end{array}\right)$.

From Proposition 1. we see that the square roots of the upper left entries of $h^{\prime}\left(m_{1} m_{1}\right), h^{\prime}\left(m_{2} m_{1} m_{1} m_{2}\right), h^{\prime}\left(m_{3} m_{2} m_{1} m_{1} m_{2} m_{3}\right)$, etc. satisfy a Euclidean algorithm sequence (in reverse order) where each quotient is either $X$ or $X+1$. Those sequences are often called maximal length sequences for the Euclidean algorithm or maximal length Euclidean sequences, and they have long been a topic of interest in number theory. Mesirov and Sweet [8] showed that, when $a \in \mathbb{F}_{2}[X]$ is irreducible, there exist exactly two polynomials $d$ such that $a, d$ are the first terms of a maximal length Euclidean sequence. They also provide an algorithm to compute them, which we will give below.

In their collision algorithm, Grassl et al. apply Mesirov and Sweet's algorithm to the irreducible polynomial $a=p(X)$ in order to recover $d$. The corresponding bit sequence $m_{1} \ldots m_{n}$ can be recovered by applying the Euclidean algorithm to $a$ and $d$. By Proposition 1] we have

$$
h^{\prime}\left(0 m_{n} \ldots m_{1} m_{1} \ldots m_{n} 0\right)=h^{\prime}\left(1 m_{n} \ldots m_{1} m_{1} \ldots m_{n} 1\right)+\left(\begin{array}{cc}
a^{2} & a^{2} \\
a^{2} & 0
\end{array}\right)
$$

hence

$$
H^{\prime}\left(0 m_{n} \ldots m_{1} m_{1} \ldots m_{n} 0\right) \equiv H^{\prime}\left(1 m_{n} \ldots m_{1} m_{1} \ldots m_{n} 1\right)
$$




\subsection{Maximal Length Sequences in the Euclidean Algorithm in $\mathbb{F}_{2}[X]$}

The Mesirov and Sweet's algorithm, as described by Grassl et al., is the following one. To find a maximal length Euclidean sequence starting from a given polynomial $a(X)$ of degree $k$,

1. Construct a matrix $A \in \mathbb{F}_{2}^{(k+1) \times k}$ from the $k+1$ polynomials

$$
\begin{aligned}
g_{0} & =1, \\
g_{i} & =X^{i-1}+X^{2 i-1}+X^{2 i} \bmod a(X), \quad \text { for } i=1,2, \ldots, k,
\end{aligned}
$$

placing in the $i^{\text {th }}$ row of $A$ the coefficients $g_{i, 0}, g_{i, 1}, \ldots, g_{i, k-1}$ of the polynomial $g_{i}(X)=g_{i, 0}+g_{i, 1} X+\ldots+g_{i, k-1} X^{k-1}$.

2. Solve the linear system $A u^{t}=(1,0, \ldots, 0,1)^{t}$ where $u=\left(u_{1}, \ldots, u_{k}\right)$.

3. Compute $d(X)$ by multiplying $a(X)$ by $\sum_{i=1}^{k} u_{i} X^{-i}$ and taking only the non-negative powers.

Mesirov and Sweet showed in 8 that polynomials $d$ such that $a, d$ are the first terms of a maximal length sequence for the Euclidean algorithm, are in oneto-one correspondence with the solutions of the equation $A u^{t}=(1,0, \ldots, 0,1)^{t}$. Moreover, they proved that when $a$ is irreducible, this equation has exactly two solutions.

Maximal length Euclidean sequences are closely connected to the matrices $A_{0}^{\prime}$ and $A_{1}^{\prime}$. If $m_{i}$ and $a_{(i)}$ are as in Proposition 1 we have

$$
\left(\begin{array}{ll}
a_{(1)} & a_{(0)}
\end{array}\right)=\left(\begin{array}{lll}
1 & 0
\end{array}\right)\left(\begin{array}{ccc}
X+1+m_{1} & 1 \\
1 & 0
\end{array}\right)=\left(\begin{array}{ll}
1 & 0
\end{array}\right) A_{\overline{m_{1}}}^{\prime}
$$

(where $\overline{m_{1}}:=1-m_{1}$ ) and for $1<i \leq k$ we have

$$
\left(a_{(i)} a_{(i-1)}\right)=\left(\begin{array}{ll}
a_{(i-1)} & a_{(i-2)}
\end{array}\right)\left(\begin{array}{cc}
X+m_{i} & 1 \\
1 & 0
\end{array}\right)=\left(a_{(i-1)} a_{(i-2)}\right) A_{m_{i}}^{\prime} .
$$

Therefore, the first row of any product of $A_{0}^{\prime}$ and $A_{1}^{\prime}$ is the beginning of a maximal length Euclidean sequence. By induction, we have

$$
\left(a_{(k)} a_{(k-1)}\right)=\left(\begin{array}{ll}
1 & 0
\end{array}\right) h^{\prime}\left(\overline{m_{1}} m_{2} \ldots m_{k}\right) .
$$

\subsection{Ideas behind Our Algorithms}

Before going into the details of our algorithms, we provide some intuition behind them. Let $m$ be the palindromic message used in Grassl et al.'s collision attack. In Section 3, we first observe that the hash values of $m 0$ and $0 m$ have the form $L:=\left(\begin{array}{cc}1 & 0 \\ \alpha & 1\end{array}\right)$ and $U:=\left(\begin{array}{cc}1 & \alpha \\ 0 & 1\end{array}\right)$ for some $\alpha \in \mathbb{F}_{2^{n}}$. Since $L$ and $U$ have order 2 , we obtain an algorithm finding preimages to the identity matrix, hence a second preimage algorithm for the Tillich-Zémor hash function.

We then observe that the set of matrices $\mathcal{L}:=\left\{L_{\alpha}:=\left(\begin{array}{cc}1 & 0 \\ \alpha & 1\end{array}\right), \alpha \in K\right\}$ forms an Abelian subgroup of $S L(2, K)$ that is isomorphic to the additive group $(K,+)$. Finding $n$ matrices $L_{\alpha_{i}}$ (together with their preimages) such that the set $\left\{\alpha_{i}, i=\right.$ 
$1, \ldots, n\}$ is a basis of $K$ over $\mathbb{F}_{2}$, therefore suffices to generate the whole subgroup $\mathcal{L}$. The same holds for the set $\mathcal{U}:=\left\{U_{\alpha}:=\left(\begin{array}{cc}1 & \alpha \\ 0 & 1\end{array}\right), \alpha \in K\right\}$. Moreover, inspired by previous work on a similar function [9], we prove in Proposition 3 that any matrix of $S L(2, K)$ can be written as a small product of $A_{0}$ and matrices of the sets $\mathcal{L}$ and $\mathcal{U}$. At this point, it remains to obtain $n$ matrices $L_{\alpha_{i}}$ and $U_{\alpha_{i}}$ generating $\mathcal{L}$ and $\mathcal{U}$. We solve this problem in two different ways and obtain two algorithms, each of them having its own advantages but both of them being very efficient and successful.

As observed above, Grassl et al.'s paper indirectly provides one matrix $L \in \mathcal{L}$ after applying Mesirov and Sweet's algorithm to $a=p$. In our first precomputing algorithm, we obtain $n$ different matrices after applying Mesirov and Sweet's algorithm to $a_{i}=p p_{i}^{\prime}$, where $p_{i}^{\prime}$ are randomly-chosen small degree polynomials. This idea is quite simple but proving its correctness requires solving two issues. First, Mesirov and Sweet only guarantee the success of their algorithm when applied to an irreducible polynomial. Second, the $\alpha_{i}$ values obtained by this way should not be restricted to any vectorial subspace of $K$. In this paper, we extend Mesirov and Sweet's result in Proposition 5 and then argue on the correctness of our algorithm.

In our second precomputing algorithm, we follow a different approach and obtain $n$ matrices recursively from the first one. In particular, we exhibit a sequence of messages with increasing lengths hashing to matrices of the required form, such that the corresponding values $\alpha_{i}$ satisfy a very simple recurrence. By studying the elements of this recurrence, we identify a subset $I \subset\{1, \ldots, 2 n\}$ such that the corresponding matrices $\left\{L_{\alpha_{i}}, i \in I\right\}$ generate the whole subgroup when $n$ is prime. This important result is proved through Lemma 6. Lemma 7 and Proposition 9. Matrices $\left\{U_{\alpha_{i}}, i \in I\right\}$ and their preimages are recovered at the same time.

Due to the way it constructs matrices $L_{\alpha_{i}}$ and $U_{\alpha_{i}}$, our second precomputing algorithm produces larger preimages than the first one. On the other hand, it is deterministic, faster than the first one, and it is guaranteed to always succeed when parameter $n$ is prime.

\section{Second Preimages for Tillich-Zémor Hash Function}

The following proposition constructs collisions with the void message from the palindromic messages used in Grassl et al.'s attack.

Proposition 2. Let $\left(\begin{array}{cc}a^{2} & b \\ b & d^{2}\end{array}\right)=H^{\prime}(m)$ with $a \equiv 0$ be the modified Tillich-Zémor hash value of some message $m \in\{0,1\}^{*}$. Then

$$
H(0 m 0 m)=H(1 m 1 m)=H(m 0 m 0)=H(m 1 m 1)=I=H() .
$$

Proof: We have $1=\operatorname{det}\left(h^{\prime}(m)\right)=a^{2} d^{2}+b^{2} \equiv b^{2}$ hence $b \equiv 1$. By a straightforward computation, we have $H^{\prime}(0 m)=\left(\begin{array}{cc}1 & X+d^{2} \\ 0 & 1\end{array}\right), H^{\prime}(m 0)=\left(\begin{array}{cc}1 & 0 \\ X+d^{2} & 1\end{array}\right), H^{\prime}(1 m)=$ $\left(\begin{array}{ll}1 & X+1+d^{2} \\ 0 & 1\end{array}\right), H^{\prime}(m 1)=\left(\begin{array}{cc}1 & 0 \\ X+1+d^{2} & 1\end{array}\right)$, and all these matrices have order 2 . Finally, we observe that for any $\tilde{m} \in\{0,1\}^{*}$ such that $H^{\prime}(\tilde{m})=I$, we have 
$H(\tilde{m})=A_{0} H^{\prime}(\tilde{m}) A_{0}^{-1}=A_{0} A_{0}^{-1}=I$.

The message $m$ in Proposition 2 can be obtained by applying Mesirov and Sweet's algorithm to $a=p(X)$ as in Grassl et al.'s attack (see Proposition 1). We therefore obtain a message $\tilde{m}$ colliding with the void message for the TillichZémor hash function. A second preimage algorithm is straightforwardly deduced, since for any $m \in\{0,1\}^{*}$ we have $H(m \tilde{m})=H(m)$.

\section{Preimage Algorithm from a Few Precomputed Preimages}

For the remaining of the paper, we define $L_{\alpha}:=\left(\begin{array}{ll}1 & 0 \\ \alpha & 1\end{array}\right)$ and $U_{\beta}:=\left(\begin{array}{ll}1 & \beta \\ 0 & 1\end{array}\right)$ for any $\alpha, \beta \in K$. In [9], preimages for the LPS hash function (a function similar to Tillich-Zémor, with different matrix generators) were computed by decomposing any matrix into a product of generators and diagonal matrices, a subset of matrices for which computing preimages appeared to be easier. In the case of Tillich-Zémor, the proof of Proposition 2 suggests the following decomposition.

Proposition 3. Given a preimage of length at most $L$ for every matrix among a set $\mathcal{S}=\left\{L_{\alpha_{i}}, U_{\beta_{i}}, i=1, \ldots, n\right\}$ where $\left\{\alpha_{i}, i=1, \ldots, n\right\}$ and $\left\{\beta_{i}, i=1, \ldots, n\right\}$ are two basis of $K$ as a vector space over $\mathbb{F}_{2}$, there is a deterministic algorithm computing preimages of length at most $3 n L+5$ for the Tillich-Zémor hash function, in time $O\left(n^{3}\right)$.

PROOF: We first observe that it is sufficient to have an algorithm with the same characteristics for the modified Tillich-Zémor hash function. Indeed, for any $A, B, C, D$ with $A D+B C=1$,

$$
H(m)=\left(\begin{array}{cc}
A & B \\
C & D
\end{array}\right) \Leftrightarrow H^{\prime}(m)=A_{0}^{-1}\left(\begin{array}{cc}
A & B \\
D
\end{array}\right) A_{0} .
$$

Now, suppose we are given $M=(\underset{C}{A} \underset{D}{A})$ with $\operatorname{det}(M)=1$ and we want to find a preimage of $M$ for the modified Tillich-Zémor function. If $B \neq 0$, it is easily checked that

$$
\left(\begin{array}{ll}
A & B \\
C & D
\end{array}\right)=\left(\begin{array}{ll}
1 & 0 \\
\alpha & 1
\end{array}\right)\left(\begin{array}{cc}
X & 1 \\
1 & 0
\end{array}\right)\left(\begin{array}{ll}
1 & \beta \\
0 & 1
\end{array}\right)\left(\begin{array}{cc}
X & 1 \\
1 & 0
\end{array}\right)^{3}\left(\begin{array}{ll}
1 & 0 \\
\gamma & 1
\end{array}\right)
$$

with

$$
\left\{\begin{array}{l}
\alpha=(D X+X+B) /(X B) \\
\beta=\left(B+X^{3}\right) / X^{2} \\
\gamma=\left(X+B+X^{2} B+A X\right) /(X B)
\end{array}\right.
$$

while if $B=0$, we have

$$
\left(\begin{array}{ll}
A & 0 \\
C & D
\end{array}\right)=\left(\begin{array}{ll}
X & 1 \\
1 & 0
\end{array}\right)\left(\begin{array}{cc}
C & D \\
A+C X & D X
\end{array}\right)
$$

and $D \neq 0$, so we may apply the above decomposition to the last matrix. 
Since $\left\{\alpha_{i}, i=1, \ldots, n\right\}$ and $\left\{\beta_{i}, i=1, \ldots, n\right\}$ are two basis of $K$, we may write $\alpha=\sum_{i \in I_{\alpha}} \alpha_{i}, \beta=\sum_{i \in I_{\beta}} \beta_{i}$ and $\gamma=\sum_{i \in I_{\gamma}} \alpha_{i}$, for some $I_{\alpha}, I_{\beta}, I_{\gamma} \subset$ $\{1, \ldots, n\}$. Moreover, those decompositions can be recovered in time $O\left(n^{3}\right)$ by solving three corresponding linear systems over $\mathbb{F}_{2}$. Finally, we observe that for any $I \subset\{1, \ldots, n\}$, we have

$$
\left(\begin{array}{cc}
1 & 0 \\
\sum_{i \in I} \alpha_{i} & 1
\end{array}\right)=\prod_{i \in I}\left(\begin{array}{cc}
1 & 0 \\
\alpha_{i} & 1
\end{array}\right) \quad \text { and } \quad\left(\begin{array}{cc}
1 & \sum_{i \in I} \beta_{i} \\
0 & 1
\end{array}\right)=\prod_{i \in I}\left(\begin{array}{cc}
1 & \beta_{i} \\
0 & 1
\end{array}\right) .
$$

Putting together what we have seen so far, we obtain a decomposition of any matrix into at most 5 matrices $A_{0}^{\prime}$ and $3 n$ matrices from the set $\mathcal{S}$. A preimage is obtained by concatenating the preimages of the corresponding matrices, and the maximal length of this preimage follows.

Let $\mu_{L}, \mu_{U}:\{0,1\}^{*} \rightarrow\{0,1\}^{*}$ be two transformations on bitstrings defined as follows:

$$
\begin{aligned}
& \mu_{L}\left(m_{1} m_{2} \ldots m_{k}\right)=m_{k} \ldots m_{2} \overline{m_{1} m_{1}} m_{2} \ldots m_{k} 0 \\
& \mu_{U}\left(m_{1} m_{2} \ldots m_{k}\right)=0 m_{k} \ldots m_{2} \overline{m_{1} m_{1}} m_{2} \ldots m_{k} .
\end{aligned}
$$

Lemma 4. Let $m \in\{0,1\}^{*}$ such that $\left(\begin{array}{ll}1 & 0\end{array}\right) H^{\prime}(m)=\left(\begin{array}{ll}0 & q\end{array}\right)$ for some $q \in K$. Then

$$
H^{\prime}\left(\mu_{L}(m)\right)=\left(\begin{array}{cc}
1 & 0 \\
X+q^{2} & 1
\end{array}\right) \quad \text { and } \quad H^{\prime}\left(\mu_{U}(m)\right)=\left(\begin{array}{cc}
1 & X+q^{2} \\
0 & 1
\end{array}\right) .
$$

Proof: Let $m=m_{1} \ldots m_{k}$ be the bitwise representation of $m$. According to Equation 1 and Proposition 1 we have $H^{\prime}\left(m_{k} \ldots m_{2} \bar{m}_{1} \bar{m}_{1} m_{2} \ldots m_{k}\right)=\left(\begin{array}{cc}0 & b \\ b & q^{2}\end{array}\right)$. Moreover $b=1$ since the determinant of any hash value is 1 . Multiplying left and right by $A_{0}$ we obtain the result.

Proposition 3 reduces the preimage problem to the problem of precomputing the preimages of some set of matrices. Lemma 4 further reduces the precomputation to find $n$ messages $m_{i}, i=1, \ldots, n$ such that

$$
\left(\begin{array}{ll}
1 & 0
\end{array}\right) H^{\prime}\left(m_{i}\right)=\left(\begin{array}{ll}
0 & q_{i}
\end{array}\right) \text { for some } q_{i} \in K
$$

and $\left\{q_{i}^{2}+X \bmod p, i=1, \ldots, n\right\}$ is a basis of $K$. In Sections 5 and 6 , we give two algorithms for finding these messages.

\section{First Precomputing Algorithm}

As observed in Section 3, we can obtain one message satisfying Equation 2 by applying Mesirov and Sweet's algorithm to $a=p$. In order to obtain more messages satisfying the equation, a natural idea is to apply the algorithm to a small multiple of $p$. This leads us to the following algorithm. 
1. Take $R$ large enough.

2. Construct a set $T=\left\{\alpha_{i}, i=1, \ldots, n\right\}$ containing elements of $K$ that are linearly independent over $\mathbb{F}_{2}$, as well as preimages to $L_{\alpha_{i}}$ and $U_{\alpha_{i}}$. To this aim, start from an empty set $T$, then until the set contains $n$ elements:

(a) Generate a random irreducible polynomial $p^{\prime}$ of degree $R$.

(b) Construct a matrix $A$ by applying the first step of Mesirov and Sweet's algorithm to $a=p p^{\prime}$.

(c) If $A u^{t}=(1,0, \ldots, 0,1)^{t}$ has solutions, compute $\alpha:=d^{2}+X$ where $d$ is obtained by completing Mesirov and Sweet's algorithm.

(d) Check whether $\alpha$ is independent of the elements of $T$; if it is, add it to the list and compute the corresponding preimages.

The algorithm is conceptually simple but it is not a trivial task to prove its correctness. First, Mesirov and Sweet only guarantee the success of their algorithm for irreducible polynomials while in Step 2(b) we apply it to a more general polynomial. Second, the above algorithm succeeds only if it is possible to generate $n$ independent $\alpha_{i}$ values in Step 2(d). This last condition seems particularly hard to prove given our current understanding of maximal length Euclidean sequences in $\mathbb{F}_{2^{n}}$.

In Section 5.1 below, we extend Mesirov and Sweet's result and argue that in Step 2(c) of our algorithm, the system $A u^{t}=(1,0, \ldots, 0,1)^{t}$ has solutions with a probability $\rho$ at least $1 / 2$ on average. In Section 5.2 we provide intuitive arguments and experimental evidence showing that if $R$ is $O(\log n)$, the $\operatorname{loop}$ of Step 2 must only be repeated $O(n)$ times. Since each loop requires solving at most two linear systems of size $n$, we expect the whole algorithm to run in probabilistic $O\left(n^{4}\right)$ time.

All the messages constructed by this algorithm have length exactly $R+n$. These messages can be used in Lemma 4 and Proposition 3 to compute preimages of length $O\left(n^{2}+n R\right) \approx O\left(n^{2}\right)$ for any matrix.

\subsection{Mesirov and Sweet's Algorithm for $a=p p^{\prime}$}

In this section, we argue that for a parameter $p$ chosen at random, the probability that $A u^{t}=(1,0, \ldots, 0,1)^{t}$ has solutions in Step $2(\mathrm{c})$ of our algorithm is $\rho \approx 1 / 2$. Let us consider the arithmetic sequence

$$
1+p+\ell X(X+1) p, \quad \ell \in \mathbb{F}_{2}[X], \quad \operatorname{deg}(\ell) \leq R-1 .
$$

For any $\ell \in \mathbb{F}_{2}[X]$, let $N(\ell)$ be the number of distinct irreducible polynomials of degree $R$ in the factorization of $1+p+\ell X(X+1) p$. Let $N_{0}:=\sum_{\operatorname{deg}(\ell) \leq R-2} N(\ell)$ and $N_{1}:=\sum_{\operatorname{deg}(\ell)=R-1} N(\ell)$. Although a priori there may exist some $R$ and $p$ such that $N_{0} \approx 0$, for most values $R$ and random polynomials $p$ it seems reasonable to expect $N_{0} \approx N_{1}$. In the following, we show that the probability that $A u^{t}=(1,0, \ldots, 0,1)^{t}$ has solutions is at least $\frac{N_{0}}{N_{0}+N_{1}}$, hence for a parameter $p$ chosen at random we expect to have $\rho$ at least equal to $1 / 2$.

By simple linear algebra the system $A u^{t}=(1,0, \ldots, 0,1)^{t}$ has solutions if both 1 ) the first row of $A$ is a linear combination of its last row and some other rows 
and 2) the last row of $A$ is not a linear combination of its middle rows. In other terms,

1. There exists $v=\left(v_{0}, \ldots, v_{n+R}\right) \in \mathbb{F}_{2}^{(n+R+1) \times 1}$ such that $v A=0$ and $v_{0}=$ $v_{n+R}=1$.

2. For any $w=\left(w_{0}, \ldots, w_{n+R}\right) \in \mathbb{F}_{2}^{(n+R+1) \times 1}$ such that $w A=0$ and $w_{0}=0$, we have $w_{n+R}=0$.

Following Mesirov and Sweet [8], let us consider the following equations in the (polynomial) variables $r(X)$ and $s(X)$ :

$$
\begin{aligned}
r(X)+X r(X)^{2}+X^{2} r(X)^{2} & =1 \bmod a(X), \\
s(X)+X s(X)^{2}+X^{2} s(X)^{2} & =0 \bmod a(X) .
\end{aligned}
$$

Remembering the definition of $A$, we see that $A u^{t}=(1,0, \ldots, 0,1)^{t}$ has solutions if

1. For some $r(X)=r_{n+R-1} X^{n+R-1}+\ldots+r_{1} X+r_{0}$ solution to Equation 3 , we have $r_{n+R-1}=1$.

2. For any $s(X)=s_{n+R-1} X^{n+R-1}+\ldots+s_{1} X+s_{0}$ solution to Equation 4, we have $s_{n+R-1}=0$.

Since $X$ does not divide $a=p p^{\prime}$, it must divide $a+1$. The polynomial $(a+1) / X$ has degree $n+R-1$ and is a solution to Equation 3, it therefore satisfies the first condition. Equation 4 has four solutions $s_{00}, s_{01}, s_{10}$ and $s_{11}$ characterized as follows:

$$
\begin{array}{ll}
\left\{\begin{array}{l}
s_{00}=0 \bmod p, \\
s_{00}=0 \bmod p^{\prime} ;
\end{array}\right. & \left\{\begin{array}{l}
s_{10}=0 \bmod p, \\
1+X(X+1) s_{10}=0 \bmod p^{\prime} ;
\end{array}\right. \\
\left\{\begin{array}{l}
1+X(X+1) s_{01}=0 \bmod p, \\
s_{01}=0 \bmod p^{\prime} ;
\end{array}\right. & \left\{\begin{array}{l}
1+X(X+1) s_{11}=0 \bmod p, \\
1+X(X+1) s_{11}=0 \bmod p^{\prime} .
\end{array}\right.
\end{array}
$$

Clearly, $s_{00}=0 \bmod a$. Since $X+1$ does not divide $a=p p^{\prime}$, it must divide $a+1$. The polynomial $(a+1) / X(X+1)$ has degree $n+R-2$ and is a solution to Equation 4, Reducing it modulo $p$ and $p^{\prime}$, we see that $(a+1) / X(X+1)=s_{11}$. As Equation 4 is homogeneous, its solutions form a vector space, hence $s_{01}=$ $s_{10}+s_{11}$. Since the coefficient $n+R-1$ of $s_{11}$ is zero, the coefficient $N+R-1$ of $s_{01}$ and $s_{10}$ are equal. Using Chinese Remainder Theorem, we have

$$
s_{10}=\left[\left(X(X+1) p^{\prime}\right)^{-1} \bmod p\right] p^{\prime}
$$

hence the coefficient $N+R-1$ of $s_{10}$ is equal to the coefficient $N-1$ of $\left(X(X+1) p^{\prime}\right)^{-1} \bmod p$. We have proved the following proposition that extends Mesirov and Sweet's result on irreducible polynomials to polynomials with two distinct nonlinear irreducible factors: 
Proposition 5. Let $p, p^{\prime}$ be nonlinear irreducible polynomials and let $a=p p^{\prime}$. If

$$
\operatorname{deg}\left(\left[X(X+1) p^{\prime}\right]^{-1} \bmod p\right) \leq \operatorname{deg}(p)-2 .
$$

then the corresponding Mesirov and Sweet system $A u^{t}=(1,0, \ldots, 0,1)^{t}$ has solutions.

Let $y:=\left[X(X+1) p^{\prime}\right]^{-1} \bmod p$. By definition, we have

$$
y X(X+1) p^{\prime}=1+k p
$$

for some unique $k \in \mathbb{F}_{2}[X]$. As $\operatorname{deg}(y) \leq N-1$, we have $\operatorname{deg}(k) \leq R+1$. We easily see that $X(X+1)$ divides $1+k p$ if and only if $k=1+X(X+1) \ell$ for some $\ell \in \mathbb{F}_{2}[X]$ with $\operatorname{deg}(\ell) \leq R-1$. Therefore for any $p^{\prime}$ (randomly generated in our algorithm), there exists exactly one polynomial $\ell$ with $\operatorname{deg}(\ell) \leq R-1$ such that

$$
y X(X+1) p^{\prime}=1+p+X(X+1) \ell p .
$$

If $\ell \neq 0$, then by Proposition [5] the system $A u^{t}=(1,0, \ldots, 0,1)^{t}$ has solutions if $\operatorname{deg}(\ell)=\operatorname{deg}(y)+R-\operatorname{deg}(p) \leq R-2$. For $\ell=0$ we have $\operatorname{deg}(y)=\operatorname{deg}(p)-2-R$ which satisfies the condition of Proposition 5. Defining $N_{0}$ and $N_{1}$ as above, $N_{0}$ (respectively $N_{1}$ ) is precisely the number of irreducible polynomials $p^{\prime}$ of degree $R$ such that the corresponding polynomial $\ell$ satisfies $\operatorname{deg}(\ell) \leq R-2$ (respectively $\operatorname{deg}(\ell)=R-1)$. We finally obtain $\rho \geq \frac{N_{0}}{N_{0}+N_{1}}$.

Remark. It is possible to remove the irreducibility condition on $p^{\prime}$ in Step 2(a) of our algorithm. However, the probability that Mesirov and Sweet system $A u^{t}=$ $(1,0, \ldots, 0,1)^{t}$ has solutions in Step 2(c) is maximal when $p^{\prime}$ is irreducible, as can be seen by further extending Proposition 5 .

\subsection{Correctness of the Algorithm}

Even after finding many different $\alpha$ values in Step 2(c), our algorithm could still fail if all these values belonged to a vector subspace of $K$. However, the algorithm will always succeed if the following hypothesis holds.

Hypothesis 1. The polynomials $d$ resulting from applying the Mesirov and Sweet's algorithm to $a=p p^{\prime}$ (with $p$ and $p^{\prime}$ irreducible, $p$ fixed and $p^{\prime}$ random), form a set without any particular structure. In particular, this set can be thought of as a random set of polynomials of degree $n+R-1$.

If each $d$ value constructed can be considered as a random polynomial of degree $n+R-1$, then the corresponding value $\alpha:=d^{2}+X \bmod p$ can be considered as a random polynomial of degree at most $n-1$. In that case, every new value $\alpha$ is (very) likely to be independent from the previous one: if there are already $i$ independent elements in $T$, the new value will be independent from the previous ones with a probability $1-2^{i-n}$. After generating a little more than $n$ polynomials $d$, our algorithm will be likely to succeed in finding $n$ linearly independent 
$\alpha$ values. By the analogue of the prime number theorem for irreducible polynomials over a finite field, the number of irreducible polynomials $p^{\prime}$ of degree $R$ is roughly $2^{R} / R$. By our analysis in Section 5.1. the Mesirov and Sweet's system has solutions for at least one half of the corresponding polynomials $a=p p^{\prime}$. Therefore, we must have

$$
\frac{2^{R}}{R} \geq 2 n
$$

Assuming Hypothesis 1 is correct, taking a value $O(\log n)$ for $R$ in Step 1 should be sufficient to guarantee the success of our algorithm.

Hypothesis 1 seems very likely to hold. Of course, there is a strong relationship between two polynomials $a$ and $d$ such that all their partial quotients are $X$ or $X+1$. However, this relation does not seem to restrict $d$ to any vector subspace of $K$, even if $a$ is only chosen among the multiples of $p$. Today, the "simplest" relation known to hold between two consecutive terms of a maximal length Euclidean sequence is precisely given by the Mesirov and Sweet's algorithm, and it does not seem to impose such a restriction.

A theoretical analysis of our algorithm based on firmer grounds than Hypothesis 1 would be very valuable, but given current understanding of maximal length Euclidean sequences in $\mathbb{F}_{2^{n}}$, we believe that it is far from reach. To prove the efficiency of our algorithm, we therefore complete our arguments with experimental results. In our experiments, we took $p$ the shortest irreducible polynomial of degree $n$, that is the polynomial $p=X^{n}+\ldots+p_{0}$ for which $\sum p_{i} 2^{i}$ is minimal. For some values of $n$ between 5 and 2039, we tried different values of $R$ until we found one that was large enough. The results are given in Figure 1.

\begin{tabular}{|c|c||c|c||c|c|}
\hline$n$ & $R$ & $n$ & $R$ & $n$ & $R$ \\
\hline 5 & 5 & 67 & 11 & 257 & 13 \\
7 & 7 & 73 & 11 & 277 & 13 \\
11 & 8 & 83 & 11 & 307 & 13 \\
13 & 9 & 97 & 11 & 331 & 14 \\
17 & 9 & 103 & 12 & 353 & 14 \\
19 & 9 & 109 & 12 & 379 & 14 \\
23 & 9 & 127 & 12 & 401 & 14 \\
29 & 9 & 137 & 12 & 449 & 14 \\
31 & 10 & 149 & 12 & 499 & 14 \\
37 & 10 & 167 & 13 & 607 & 15 \\
41 & 10 & 191 & 13 & 1021 & 15 \\
47 & 10 & 211 & 13 & 2039 & 16 \\
59 & 11 & 233 & 13 & \multicolumn{1}{|c|}{} \\
\hline
\end{tabular}

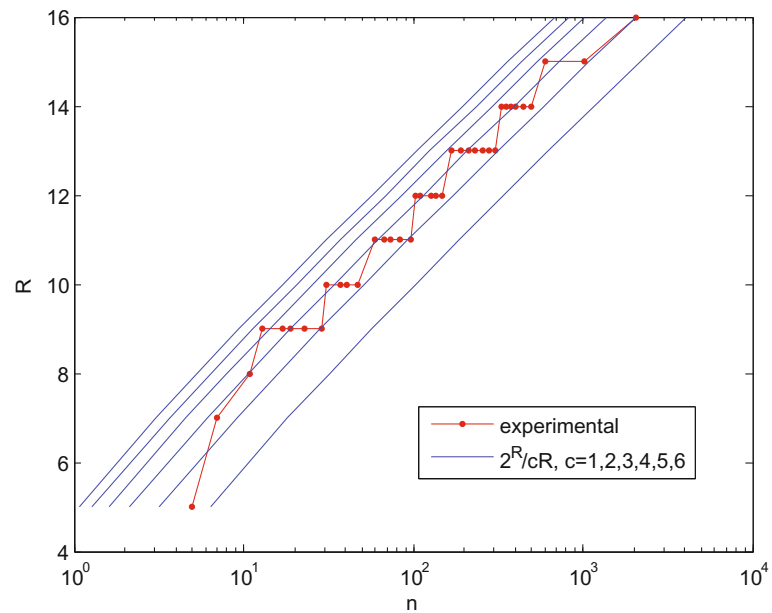

Fig. 1. Minimal value $R$ for different values $n$ and the "shortest" polynomials of degree $n$. The points on the staircase-like curve are experimental results. The other curves are $n=\frac{2^{R}}{c R}$ for $c=1,2,3,4,5,6$. 
The algorithm always succeeded with a value $R$ satisfying

$$
\frac{2^{R}}{R} \geq 5 n
$$

that is slightly larger than predicted by Equation 5 but still consistent with the expected $R=O(\log n)$. When $n \geq 17$, it always succeeded as long as $\frac{2^{R}}{R} \geq 4 n$. The last points of Figure 1 for $n=1021$ and $n=2039$ are very close to the bound of Equation 5. The results confirm the analysis performed in this section and in the previous one. In Appendix B, we describe additional experiments performed on randomly chosen polynomials. All the results obtained are also consistent with our analysis.

\section{Second Precomputing Algorithm}

Our first precomputing algorithm is very efficient both in theory and in practice, but its correctness must likely rely on some ad hoc hypothesis. In this section, we provide an alternative algorithm precomputing messages of length bounded by $n^{2}$, resulting in preimages of length $O\left(n^{3}\right)$ after applying Lemma 4 and Proposition 3. On the one hand this second algorithm is worse than the first one since it produces larger preimages, but on the other hand it is deterministic and it runs in time $O\left(n^{3}\right)$, much faster than the first one. More importantly from a theoretical point of view, we provide a proof that it always succeeds when $n$ is prime, and strong evidence that it has a very large probability of success when $n$ is reasonably large and the polynomial $p$ is chosen at random.

The Mesirov and Sweet's algorithm applied to $p$ is guaranteed to succeed since $p$ is irreducible. It provides a polynomial $q$ of degree $n-1$ such that $p$ and $q$ are the first terms of a maximal length Euclidean sequence. By Equation 1, we have $(p q)=\left(\begin{array}{ll}1 & 0\end{array}\right) h^{\prime}\left(\tilde{m}_{1}\right)$ for some $\tilde{m}_{1} \in\{0,1\}^{n}$ that can be recovered with the Euclidean algorithm. For $i>1$, let

$$
\tilde{m}_{i}:=\tilde{m}_{i-1} 0 \tilde{m}_{1}
$$

We first show that the messages $\tilde{m}_{i}$ satisfy the requirements of Lemma 4 .

Lemma 6. For any $i \geq 0,\left(\begin{array}{ll}1 & 0\end{array}\right) H^{\prime}\left(\tilde{m}_{i}\right) \equiv\left(\begin{array}{ll}0 & q^{i}\end{array}\right)$.

Proof: For $i=1$ the result is trivial. Moreover, assuming the property is satisfied for some $i$, it is also satisfied for $i+1$ since

$$
\begin{aligned}
\left(\begin{array}{ll}
1 & 0
\end{array}\right) H^{\prime}\left(\tilde{m}_{i+1}\right) & =\left(\begin{array}{ll}
1 & 0
\end{array}\right) H^{\prime}\left(\tilde{m}_{i}\right) A_{0}^{\prime} H^{\prime}\left(\tilde{m}_{1}\right) \\
& \equiv\left(\begin{array}{ll}
0 & q^{i}
\end{array}\right)\left(\begin{array}{cc}
X & 1 \\
1 & 0
\end{array}\right)\left(\begin{array}{cc}
0 & q \\
\ldots & \ldots
\end{array}\right) \equiv\left(\begin{array}{ll}
q^{i} & 0
\end{array}\right)\left(\begin{array}{cc}
0 & q \\
\ldots & \ldots
\end{array}\right) \equiv\left(\begin{array}{ll}
0 & q^{i+1}
\end{array}\right) .
\end{aligned}
$$

To apply Lemma 4 and Proposition 3 , we must find a subset of $\left\{q^{2 i}+X \bmod \right.$ $p, i \geq 1\}$ that is a basis of $K$. We start by studying the sets $S_{j}:=\left\{q^{2(j+i)} \bmod \right.$ $p, i=1, \ldots, n\}$. 
Lemma 7. If $n$ is prime, then for any $j \geq 0$, the set $S_{j}:=\left\{q^{2(j+i)} \bmod p, i=\right.$ $1, \ldots, n\}$ is a basis of $K$ over $\mathbb{F}_{2}$.

Proof: It suffices to show that each $S_{j}$ is a free set of $K$. Let us assume by contradiction that there exists $\left(b_{1}, \ldots, b_{n}\right) \in \mathbb{F}_{2}^{n} \backslash\{(0, \ldots, 0)\}$ such that $0=$ $\sum_{i=1}^{n} b_{i} q^{2(j+i)}$. Since $q$ is a polynomial of degree $n-1, q \neq 0,1$ hence $q^{2} \neq 0,1$. The degree of the minimal polynomial of $q^{2}$ must divide $n$, hence if $n$ is prime it is either 1 or $n$. Since $q^{2} \neq 0,1$ the minimal polynomial degree has degree larger than 1 and hence it has degree $n$. Now, let us define the polynomial $f: y \rightarrow f(y)=\sum_{i=0}^{n-1} b_{i-1} y^{i}$. We have $\operatorname{deg}(f)<n$. We also have $q^{2(j+1)} f\left(q^{2}\right)=0$ hence $f\left(q^{2}\right)=0$. Therefore $f$ must be a multiple of the minimal polynomial of $q^{2}$, which brings us to a contradiction.

The proof of Lemma 7 does not work if $n$ is not prime since the degree of the minimal polynomial of $q^{2}$ may then be a nontrivial divisor of $n$. However, even if $n$ is not prime, $S_{j}$ is a basis for all $j$ if and only if $S_{j}$ is a basis for some $j$. If $n_{1}$ is a divisor of $n$, the probability that a random element of $K$ has a minimal polynomial with degree dividing $n_{1}$ is $2^{n_{1}-n}$, that is very small for reasonably large values of $n$ (Tillich and Zémor suggested $n>130$ ). Of course, $q^{2}$ is not a random element of $K$ since $q$ is one of the two polynomials making $p$ and $q$ the first terms of a maximal length Euclidean sequence. However, the best link known today between $p$ and $q$ is the Mesirov and Sweet's algorithm, and when $p$ is chosen at random this algorithm does not seem to influence the degree of the minimal polynomial of $q$. We have confirmed this intuition experimentally: for large $n$ and random $p, S_{0}$ was always a basis, while for very small values of $n$ like $n=4$ it was not always (although most often) the case 1 .

Let $T_{j}:=\left\{q^{2(i+j)}+X \bmod p, i \geq 1\right\}$. We now show that if $S_{0}$ is a basis over $K$, then there exists $j \leq n$ such that $T_{j}$ is a basis of $K$. To this aim we first need the following lemma.

Lemma 8. Let $\left\{\beta_{i}, i=1, \ldots, n\right\}$ be a basis of $K$ over $\mathbb{F}_{2}$ and let $e_{i} \in \mathbb{F}_{2}, i=$ $1, \ldots, n$ be such that $X=\sum_{i} e_{i} \beta_{i}$. Then $\left\{\alpha_{i}:=\beta_{i}+X, i=1, \ldots, n\right\}$ is a basis of $K$ over $\mathbb{F}_{2}$ if and only if $\sum_{i} e_{i}=0$.

Proof: We can write $X=\sum_{i} e_{i} \alpha_{i}+\left(\sum_{i} e_{i}\right) X$. If $\sum_{i} e_{i}=1$, then $0=\sum_{i} e_{i} \alpha_{i}$ hence $\left\{\alpha_{i}, i=1, \ldots, n\right\}$ is not a free set of elements. On the other hand, if $\sum_{i} e_{i}=0$ then $X=\sum_{i} e_{i} \alpha_{i}$. Let $\alpha \in K$ and let $b_{i} \in \mathbb{F}_{2}, i=1, \ldots, n$ be such that $\alpha=\sum_{i} b_{i} \beta_{i}$. Let $a_{i}:=b_{i}+\left(\sum_{i} b_{i}\right) e_{i}$. We have $\alpha=\sum_{i} b_{i} \alpha_{i}+\left(\sum_{i} b_{i}\right) X=\sum_{i} a_{i} \alpha_{i}$ hence $\left\{\alpha_{i}, i=1, \ldots, n\right\}$ is a generating set.

We are now ready to prove the following result.

Proposition 9. If $S_{0}$ is a basis of $K$ over $\mathbb{F}_{2}$ (in particular, if $n$ is prime), then there exists $j<n$ such that $T_{j}$ is a basis of $K$ over $\mathbb{F}_{2}$.

Proof: We give a constructive proof of the result. Since $S_{0}$ is a basis of $K$ over $\mathbb{F}_{2}$, we know that $S_{j}$ is a basis of $K$ over $\mathbb{F}_{2}$ for all $j \geq 0$. Let $e_{i} \in \mathbb{F}_{2}, i=1, \ldots, n$

${ }^{1}$ The Tillich-Zémor hash function is a priori even weaker when $n$ is not prime, due to the subgroup structure of $S L(2, K)$ [1]. 
such that $X=\sum_{i=1}^{n} e_{i} q^{2 i}$. If $\sum_{i} e_{i}=0$, then according to Lemma 8 we are done with $j=0$. Otherwise, let $j$ be the smallest index $i$ such that $e_{i}=1$. Since $q^{2} \bmod p$ belongs to $K$, the degree $n^{\prime}$ of its minimal polynomial $p^{\prime}$ divides $n$. (This polynomial can be easily computed, but it is not needed by the algorithm.) By definition, we have $p_{0}^{\prime}+p_{1}^{\prime} q^{2}+\ldots+p_{n-1}^{\prime} q^{2\left(n^{\prime}-1\right)}+q^{2 n^{\prime}}=0 \bmod p$. Since $p^{\prime}$ is irreducible, we have $p_{0}^{\prime}=1$ (otherwise $X \mid p^{\prime}$ ) and $\sum_{i=0}^{n^{\prime}} p_{i}^{\prime}=1$ (otherwise $\left.X+1 \mid p^{\prime}\right)$. Let us define $e_{i}:=0$ for $i>n$ and $p_{i}^{\prime}:=0$ for $i>n^{\prime}$. We have

$X \equiv \sum_{i=1}^{n} e_{i} q^{2 i} \equiv \sum_{i=1}^{n} e_{i} q^{2 i}+q^{2 j}\left(1+p_{1}^{\prime} q^{2}+\ldots+p_{n^{\prime}-1}^{\prime} q^{2\left(n^{\prime}-1\right)}+q^{2 n^{\prime}}\right) \equiv \sum_{i=j+1}^{j+n}\left(e_{i}+p_{i-j}^{\prime}\right) q^{2 i}$.

Moreover, $\sum_{i=j+1}^{j+n}\left(e_{i}+p_{i-j}^{\prime}\right)=\left(1+\sum_{i=1}^{n} e_{i}\right)+\left(1+\sum_{i=0}^{n^{\prime}} p_{i}^{\prime}\right)=0$. Together with Lemma 8, this shows that $T_{j}$ is a basis of $K$ over $\mathbb{F}_{2}$.

In short, our second algorithm is as follows

1. Apply Mesirov and Sweet's algorithm to $p$; get $q$ and $\tilde{m}_{1}$.

2. If $n$ is not prime, check whether $S_{0}=\left\{q^{2}, q^{4}, \ldots, q^{2 n}\right\}$ is a basis of $K$ over $\mathbb{F}_{2}$. If it is not, abort.

3. Decompose $X$ in the basis $S_{0}$.

4. Determine $j$ as in the proof of Proposition 9.

5. Compute $\tilde{m}_{j+1}, \ldots, \tilde{m}_{j+n}$ using Equation 6 and apply Lemma 4 .

Step 1 and Step 3 both require solving a linear system of size $n \times n$ over $\mathbb{F}_{2}$ which can be done in time $O\left(n^{3}\right)$; the remaining steps are comparatively fast. The length of $\tilde{m}_{i}$ is $i(n+1)-1$ and we have $i \leq j+n$ and $j<n$. After application of the mappings $\mu_{L}$ and $\mu_{U}$, we may apply Proposition 3 with $L=4 n^{2}+2 n+1$, resulting in preimages of length $O\left(n^{3}\right)$ for any matrix. The algorithm is guaranteed to succeed if $n$ is prime, and as argued above when $n$ is composite but reasonably large it will likely succeed with a very large probability.

\section{Discussion}

In this paper, we presented very efficient algorithms computing preimages for the Tillich-Zémor hash function. We first gave a second preimage algorithm. Then we reduced the problem of finding preimages for the Tillich-Zémor hash function to the problem of precomputing a few preimages with certain properties. Subsequently, we gave two algorithms for the precomputing part:

1. The first algorithm produces messages of length $O(n)$, resulting in generic preimages of length $O\left(n^{2}\right)$. We provided theoretical and experimental evidence that it runs in probabilistic time $O\left(n^{4}\right)$ and succeeds with a very large probability on the function parameters.

2. The second algorithm produces messages of length $O\left(n^{2}\right)$, resulting in generic preimages of length $O\left(n^{3}\right)$. We gave a proof that it always succeeds when $n$ is prime, and arguments that it succeeds for most polynomials $p$ when $n$ is not necessarily prime but is reasonably large. The algorithm runs in deterministic time $O\left(n^{3}\right)$. 
Since the size of $S L\left(2, \mathbb{F}_{2^{n}}\right)$ is about $2^{3 n}$, it seems reasonable to conjecture that preimages of length $3 n$ exist for any matrix. However, even if this conjecture is true, it is not clear whether there exists an efficient algorithm computing preimages of this length. We leave that question as an interesting open problem. From a practical point of view, our first algorithm is the most interesting one since it produces shorter messages. On the other hand, the second algorithm is more appealing from a theoretical point of view. In particular, it provides a constructive proof that the Cayley graphs corresponding to the Tillich-Zémor hash function satisfy Babai's conjecture (that is, that they have a polylogarithmic diameter [7]), at least when we restrict $n$ to the prime values. Besides those important results, in the argumentation for our first algorithm we provided an extension of Mesirov and Sweet's result [8] and a connection to some arithmetic sequence of polynomials, both of which are of independent interest.

Grassl et al.'s attack found collisions for the Tillich-Zémor hash function. In this paper, we showed that the function is not even one-way. The attacks also break its vectorial and projective variants [10. Our work puts a final end to the story of the Tillich-Zémor hash function, one of the oldest and most elegant hash functions in the literature. Similar hash functions that are using the same design (replacing the group $S L(2, K)$ and the generators $A_{0}, A_{1}$ by other groups and generators) have also been cryptanalyzed recently [14 13:9].

Nevertheless, we point out that these particular attacks do not invalidate the generic design. The key tool in the cryptanalysis of Tillich-Zémor hash function is Mesirov and Sweet's algorithm which is specific to quotients $X$ and $X+1$ in the Euclidean algorithm. At the current state of knowledge, collision and preimage resistances are recovered if we replace the matrices $A_{0}$ and $A_{1}$ by $B_{0}:=\left(\begin{array}{cc}X^{2} & 1 \\ 1 & 0\end{array}\right)$ and $B_{1}:=\left(\begin{array}{cc}X+1 & 1 \\ 1 & 0\end{array}\right)$. Moreover, since current attacks do not allow controlling the form of the collisions and preimages, security might also be recovered by introducing some simple redundancy in the messages. It might even be sufficient to replace $A_{0}$ by $A_{0}^{2}$ or $A_{0}^{3}$. More generally, similar hash functions can be constructed from other non-Abelian groups and generators.

The generic design of the Tillich-Zémor hash function has many advantages over traditional hash functions like SHA: it has inherent parallelism, potentially efficient implementations in a wide range of contexts and a security equivalent to some concise mathematical problems [4]. For these reasons, we do not recommend to give it up but on the contrary, we suggest the community to look for secure and insecure instances. In the same way as many RSA instances can be insecure, especially when they are optimized for efficiency, we believe that the particularly efficient Tillich-Zémor hash function may be an unfortunately insecure instance of a more generally sound design.

Acknowledgements. We are grateful to Gilles Zémor for pointing us an error in an early version of Proposition 5. We also thank Sylvie Baudine, Franéis Koeune and Franéis-Xavier Standaert for their help in improving this paper. 


\section{References}

1. Abdukhalikov, K.S., Kim, C.: On the security of the hashing scheme based on SL2. In: Vaudenay, S. (ed.) FSE 1998. LNCS, vol. 1372, pp. 93-102. Springer, Heidelberg (1998)

2. Charles, D., Goren, E., Lauter, K.: Cryptographic hash functions from expander graphs. J. Cryptology 22(1), 93-113 (2009)

3. Charnes, C., Pieprzyk, J.: Attacking the SL2 hashing scheme. In: Safavi-Naini, R., Pieprzyk, J.P. (eds.) ASIACRYPT 1994. LNCS, vol. 917, pp. 322-330. Springer, Heidelberg (1995)

4. de Meulenaer, G., Petit, C., Quisquater, J.-J.: Hardware implementations of a variant of Zémor-Tillich hash function: Can a provably secure hash function be very efficient? (2009) (preprint)

5. Geiselmann, W.: A note on the hash function of Tillich and Zémor. In: Gollmann, D. (ed.) FSE 1996. LNCS, vol. 1039, pp. 51-52. Springer, Heidelberg (1996)

6. Grassl, M., Ilic, I., Magliveras, S., Steinwandt, R.: Cryptanalysis of the TillichZémor hash function. Cryptology ePrint Archive, Report 2009/376 (2009), http://eprint.iacr.org/

7. Helfgott, H.A.: Growth and generation in $S L_{2}(Z / p Z)$ (2005)

8. Mesirov, J.P., Sweet, M.M.: Continued fraction expansions of rational expressions with irreducible denominators in characteristic 2. Journal of Number Theory 27, 144-148 (1987)

9. Petit, C., Lauter, K., Quisquater, J.-J.: Full cryptanalysis of LPS and morgenstern hash functions. In: Ostrovsky, R., De Prisco, R., Visconti, I. (eds.) SCN 2008. LNCS, vol. 5229, pp. 263-277. Springer, Heidelberg (2008)

10. Petit, C., Quisquater, J.-J., Tillich, J.-P., Zémor, G.: Hard and easy components of collision search in the Zémor-tillich hash function: New attacks and reduced variants with equivalent security. In: Fischlin, M. (ed.) CT-RSA 2009. LNCS, vol. 5473, pp. 182-194. Springer, Heidelberg (2009)

11. Steinwandt, R., Grassl, M., Geiselmann, W., Beth, T.: Weaknesses in the $\mathrm{SL}_{2}\left(\mathbb{F}_{2^{n}}\right)$ hashing scheme. In: Bellare, M. (ed.) CRYPTO 2000. LNCS, vol. 1880, p. 287. Springer, Heidelberg (2000)

12. Tillich, J.-P., Zémor, G.: Hashing with $S L_{2}$. In: Desmedt, Y. (ed.) CRYPTO 1994. LNCS, vol. 839, pp. 40-49. Springer, Heidelberg (1994)

13. Tillich, J.-P., Zémor, G.: Collisions for the LPS expander graph hash function. In: Smart, N.P. (ed.) EUROCRYPT 2008. LNCS, vol. 4965, pp. 254-269. Springer, Heidelberg (2008)

14. Tillich, J.-P., Zémor, G.: Group-theoretic hash functions. In: Cohen, G., Lobstein, A., Zémor, G., Litsyn, S.N. (eds.) Algebraic Coding 1993. LNCS, vol. 781, pp. 90-110. Springer, Heidelberg (1994)

15. Zémor, G.: Hash functions and graphs with large girths. In: Davies, D.W. (ed.) EUROCRYPT 1991. LNCS, vol. 547, pp. 508-511. Springer, Heidelberg (1991)

16. Zémor, G.: Hash functions and Cayley graphs. Des. Codes Cryptography 4(4), 381-394 (1994)

\section{A Can We Also Compute Preimages? A Toy Example}

In this section, we provide toy examples of our algorithms. We use $n=11$ and the "smallest" irreducible polynomial of degree 11 which is $p(X)=X^{11}+X^{2}+1$. 
The representation of the sentence "Grassl et al. have shown how to find collisions. But can we also compute preimages?" in ASCII is $47726173736 \mathrm{c}$ 2065742061 6c 2e 2068617665207368 6f 77 6e 2068 6f 772074 6f 20 6669 6e 642063 6f 6c 6c 697369 6f 6e 73 2e 204275742063616 e 2077 652061 6c 73 6f 2063 6f 6d 707574652070726569 6d 61676573 3f to which corresponds the message $m_{\text {text }}=010001110111001001100001011100110111001101101100$ 001000000110010101110100001000000110000101101100001011100010000001101000011000010111011001100101 001000000111001101101000011011110111011101101110001000000110100001101111011101110010000001110100 011011110010000001100110011010010110111001100100001000000110001101101111011011000110110001101001 011100110110100101101111011011100111001100101110001000000100001001110101011101000010000001100011 011000010110111000100000011101110110010100100000011000010110110001110011011011110010000001100011 011011110110110101110000011101010111010001100101001000000111000001110010011001010110100101101101 011000010110011101100101011100110011111 . The Tillich-Zémor hash value of this message for polynomial $p(X)$ is

$$
h=\left(\begin{array}{cc}
X^{7}+X^{5}+X^{4}+X^{2}+1 & X^{8}+X^{2}+X+1 \\
X^{9}+X^{8}+X^{7}+X^{5}+X^{4}+X^{3}+X & X^{9}+X^{6}+X^{5}+X^{4}+X^{2}+X
\end{array}\right) .
$$

We compute other preimages of this matrix using the algorithms of this paper.

We start with the second preimage algorithm of Section 3 . The Mesirov and Sweet algorithm applied to $p$ gives $d(X)=X^{10}+X^{8}+X^{7}+X^{6}+X^{5}+X^{2}+X+1$ and the message $m=01011000010$. The message

$$
\tilde{m}=\mu_{L}(m) \mu_{L}(m)=[(101111001011)(11011000010) 0][(101111001011)(11011000010) 0]
$$

is a preimage of the identity matrix, hence $H\left(m_{\text {text }} \tilde{m}\right)=H\left(m_{\text {text }}\right)=h$.

We now apply our preimage algorithms to $h$. We first change the generators and we look for a preimage of

$$
h^{\prime}=\left(\begin{array}{cc}
X^{10}+X^{8}+X & X^{9}+X^{8}+X^{7}+X^{5}+X^{4}+X^{3}+X \\
X^{9}+X^{6}+X^{5}+X^{3}+X^{2} & X^{10}+X y+X^{8}+X^{7}+X^{6}+1
\end{array}\right)
$$

for the modified Tillich-Zémor hash function. We first write

$$
\left(\begin{array}{ll}
A & B \\
C & D
\end{array}\right)=\left(\begin{array}{ll}
1 & 0 \\
\alpha & 1
\end{array}\right)\left(\begin{array}{cc}
X & 1 \\
1 & 0
\end{array}\right)\left(\begin{array}{ll}
1 & \beta \\
0 & 1
\end{array}\right)\left(\begin{array}{cc}
X & 1 \\
1 & 0
\end{array}\right)^{3}\left(\begin{array}{ll}
1 & 0 \\
\gamma & 1
\end{array}\right)
$$

with

$$
\left\{\begin{array}{l}
\alpha=X^{10}+X^{9}+X^{8}+X^{7}+X^{6}+X^{5}+X^{4}+1 \\
\beta=X^{10}+X^{7}+X^{6}+X^{5}+X^{3}+X^{2}+X \\
\gamma=X^{8}+X^{4}+1
\end{array} .\right.
$$

Now, we illustrate our first precomputing algorithm. We take $R=10$ and generate random polynomials $p^{\prime}$ of degree $R$. We apply Mesirov and Sweet's algorithm to $a=p p^{\prime}$. If successful, we obtain a $d$ value and compute the corresponding $\alpha$ value. If this $\alpha$ value is linearly independent (over $\mathbb{F}_{2^{n}} / \mathbb{F}_{2}$ ) with the previous values, we keep it and compute $m$ such that $\left(\begin{array}{ll}a & d\end{array}\right)=\left(\begin{array}{ll}1 & 0\end{array}\right) H^{\prime}(m)$. The following table summarizes the results obtained. 


\begin{tabular}{|c|c|c|c|c|}
\hline 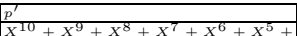 & & $\frac{\alpha}{X^{8}+X^{7}+X^{6}+X^{3}+X^{4}+X^{3}+X^{2}}$ & & \\
\hline $\mid \begin{array}{l}X+X^{3}+X^{6}+ \\
X^{4}+X^{3}+1\end{array}$ & $X^{10}+x^{9}+x^{5}+X^{4}+X$ & & & \\
\hline 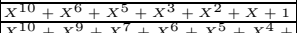 & & & & \\
\hline $\begin{array}{l}X^{10}+X^{9}+X^{7}+X^{6}+X^{5}+X^{4}+ \\
X^{3}+X^{2}+1\end{array}$ & $\begin{array}{l}X^{20}+X^{18}+X^{16}+X^{15}+X^{14}+X^{12}+ \\
X^{9}+X^{7}+X^{5}+X^{3}+X\end{array}$ & $X^{10}+X^{9}+X^{8}+X^{7}+X^{6}+X^{3}+X^{4}+1$ & yes & $m_{2}=101111101001011101100$ \\
\hline$X^{10}+X^{9}+X^{5}+X^{4}+X^{2}+X+1$ & $\begin{array}{l}X^{20}+X^{19}+X^{18}+X^{15}+X^{6}+X^{5}+ \\
X^{3}+X^{2}+X+1\end{array}$ & $X^{10}+X^{9}+X^{8}+X^{7}+X^{6}+X^{3}+X+1$ & & $m_{3}=010010111110101111100$ \\
\hline$X^{10}+X^{9}+X^{7}+X^{3}+X^{2}+X+1$ & & & & \\
\hline$X^{10}+X^{9}+X^{8}+X^{5}+1$ & $\begin{array}{l}X^{20}+X^{19}+X^{17}+X^{16}+X^{14}+X^{13}+ \\
X^{9}+X^{6}+x^{5}+X^{4}+X^{2}+1\end{array}$ & $\begin{array}{l}X^{10}+X^{9}+X^{6}+X^{5}+X^{4}+X^{3}+ \\
X^{2}+X+1\end{array}$ & ye & $m_{4}=001010011110111001001$ \\
\hline$x^{10}+x^{9}+X^{5}+x+1$ & & & & \\
\hline$\frac{X^{10}+X^{9}+X^{8}+X^{6}+X^{4}+X^{3}+1}{x^{10}+X^{1}+X^{5}+X^{3}+X^{2}+X+1}$ & & & & \\
\hline$X^{10}+X^{7}+X^{5}+X^{3}+X^{2}+X+1$ & & & & \\
\hline$\frac{X^{10}+X^{6}+X^{4}+X^{3}+1}{x^{10}+X^{9}+X^{8}+X^{4}+1}$ & & & & \\
\hline$x^{10}+x^{8}+x^{3}+x^{2}+1$ & $\begin{array}{l}X^{20}+X^{17}+X^{14}+X^{10}+X^{8}+X^{7}+ \\
X^{3}+X+1\end{array}$ & $X^{10}+X^{7}+X^{5}+X^{4}+1$ & yes & $m_{5}=010111010111101011110$ \\
\hline 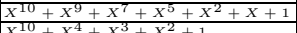 & & & & \\
\hline $\begin{array}{l}X^{10}+X^{9}+X^{8}+X^{6}+X^{5}+X^{4}+ \\
X^{3}+X^{2}+1\end{array}$ & $\begin{array}{l}X^{20}+X^{10}+X^{13}+X^{10}+X^{9}+X^{8}+ \\
X^{7}+X^{5}+X^{3}+X\end{array}$ & $X^{10}+X^{9}+X^{7}+X^{6}$ & yes & $m_{6}=100101101001110010110$ \\
\hline$x^{10}+X^{1}+X^{6}+X^{2}+1$ & & & - & \\
\hline$X^{10}+X^{8}+X^{4}+X^{3}+1$ & & & & \\
\hline$X^{10}+X^{7}+1$ & $\begin{array}{l}X^{20}+X^{18}+X^{16}+X^{14}+X^{12}+X^{11}+ \\
X^{10}+X^{7}+X^{3}+X^{2}+1\end{array}$ & $X^{9}+X^{6}+X^{5}+X^{4}+X^{3}+X$ & & $m_{7}=010010001011100010001$ \\
\hline $\begin{array}{l}X^{10}+X^{9}+X^{7}+X^{5}+X^{4}+X^{3}+ \\
X^{2}+X+1\end{array}$ & & & & \\
\hline$X^{10}+X^{8}+X^{3}+X^{2}+1$ & $\begin{array}{l}X^{20}+X^{17}+X^{14}+X^{10}+X^{8}+X^{7}+ \\
X^{3}+X+1\end{array}$ & $X^{10}+X^{7}+X^{5}+X^{4}+1$ & no & \\
\hline$\frac{X^{10}+X^{6}+X^{5}+X^{3}+X^{2}+X+1}{X^{10}+X^{8}+X^{5}+X+1}$ & $\begin{array}{l}X^{20}+X^{19}+X^{18}+X^{17}+X^{16}+X^{14}+ \\
X^{11}+X^{10}+X^{8}+X^{4}+X\end{array}$ & $X^{8}+X^{7}+X^{6}+X^{5}+X^{3}+X^{2}$ & & $m_{8}=111110110010101111001$ \\
\hline$X^{10}+X^{7}+X^{4}+X^{3}+1$ & & & & \\
\hline $\begin{array}{l}X^{10}+X^{9}+X^{7}+X^{3}+X^{4}+X^{3}+ \\
X^{2}+X+1\end{array}$ & & & & \\
\hline$X^{10}+X^{9}+X^{8}+X^{7}+1$ & $\begin{array}{l}X^{20}+X^{17}+X^{13}+X^{14}+X^{13}+X^{12}+ \\
X^{9}+X^{8}+X^{7}+X^{5}+X^{4}+X^{2}+1\end{array}$ & $X^{8}+X^{3}+X^{4}+X^{3}+X^{2}+X$ & yes & $m_{9}=100001010010010001001$ \\
\hline$\frac{X^{10}+X^{9}+X^{8}+X^{5}+X^{4}+X^{2}+1}{X^{10}+X^{9}+X^{7}+X^{6}+X^{4}+X+1}$ & $\begin{array}{l}X^{20}+X^{18}+X^{16}+X^{11}+X^{9}+X^{8}+ \\
X^{5}+X^{4}+X+1\end{array}$ & $X^{9}+X^{8}+X^{5}+X^{2}+X+1$ & & $m_{10}=101000011111000001010$ \\
\hline $\begin{array}{l}X^{10}+X^{9}+X^{8}+X^{6}+X^{5}+X^{4}+ \\
X^{3}+X^{2}+1\end{array}$ & $\begin{array}{l}X^{20}+X^{15}+X^{13}+X^{10}+X^{9}+X^{8}+ \\
X^{7}+X^{5}+X^{3}+X\end{array}$ & $X^{10}+X^{9}+X^{7}+X^{6}$ & no & \\
\hline $\begin{array}{l}X^{10}+X^{7}+X^{6}+X^{4}+X^{2}+X+1 \\
X^{10}+X^{16}+X^{6}+X^{5}+X^{2}+X\end{array}$ & & & $=$ & \\
\hline $\begin{array}{l}X^{10}+X^{7}+X^{6}+X^{3}+X^{2}+X+1 \\
X^{10}+X^{9}+X^{8}+X^{6}+X^{2}+X+1\end{array}$ & & & & \\
\hline$X^{10}+X^{9}+X^{8}+X^{7}+1$ & $\begin{array}{l}X^{20}+X^{11}+X^{10}+X^{14}+X^{13}+X^{12}+ \\
x^{9}+X^{8}+x^{7}+x^{5}+X^{4}+X^{2}+1\end{array}$ & $X^{8}+X^{5}+X^{4}+X^{3}+X^{2}+X$ & no & \\
\hline$\frac{x^{10}+x}{x^{10}+x}$ & & & - & \\
\hline$\frac{x^{10}+x^{8}+x^{7}+x^{3}+x^{3}+x^{2}+1}{x^{2}+x^{2}+x+1}$ & $\begin{array}{l}X^{20}+X^{17}+X^{10}+X^{15}+X^{14}+X^{13}+ \\
x^{11}+X^{10}+X^{9}+X^{7}+X\end{array}$ & $X^{9}+X^{7}+X^{6}+X^{5}+X^{4}+X+1$ & & $m_{11}=000011011010111000101$ \\
\hline
\end{tabular}

Writing $\alpha, \beta, \gamma$ in the basis obtained, we get $\alpha=\alpha_{2}, \beta=\alpha_{2}+\alpha_{8}+\alpha_{11}$ and $\gamma=\alpha_{1}+\alpha_{2}+\alpha_{3}+\alpha_{4}+\alpha_{5}+\alpha_{9}+\alpha_{10}$. Finally we obtain one preimage of $h$ as

where

$$
m^{\prime}=m_{\alpha} 0 m_{\beta} 000 m_{\gamma}
$$

$$
\begin{aligned}
& m_{\alpha}=\mu_{L}\left(m_{2}\right), \\
& m_{\beta}=\mu_{U}\left(m_{2}\right) \mu_{U}\left(m_{8}\right) \mu_{U}\left(m_{11}\right), \\
& m_{\gamma}=\mu_{L}\left(m_{1}\right) \mu_{L}\left(m_{2}\right) \mu_{L}\left(m_{3}\right) \mu_{L}\left(m_{4}\right) \mu_{L}\left(m_{5}\right) \mu_{L}\left(m_{9}\right) \mu_{L}\left(m_{10}\right) .
\end{aligned}
$$

(Note that the terms composing $m_{\beta}$ and $m_{\gamma}$ can be permuted arbitrarily.)

Finally, we use our second precomputing algorithm to find yet another preimage. Let $\tilde{m}_{1}=01011000010$ be the message obtained by applying Mesirov and Sweet's algorithm to $a=p$. The corresponding $q$ value is $q=d=X^{10}+X^{8}+$ $X^{7}+X^{6}+X^{5}+X^{2}+X+1$. We recursively define

$$
\tilde{m}_{i}:=m_{i-1} 0 \tilde{m}_{1} .
$$

Since $n$ is prime, we know that $S_{0}:=\left\{q^{2}, q^{4}, \cdot, q^{1+n}\right\}$ is a basis of $\mathbb{F}_{2^{n}} / \mathbb{F}_{2}$. We have $X=q^{2}+q^{4}+q^{6}+q^{8}+q^{12}+q^{18}+q^{22}$ so $T_{j}:=\left\{q^{2(i+j)}+X, i=1, \ldots, n\right\}$ is a basis for $j=1$. Let $\alpha_{i}=q^{2(j+1)}+X$ for $i=1, \ldots, n$.

Writing $\alpha, \beta, \gamma$ in this basis, we get $\alpha=\alpha_{1}+\alpha_{4}+\alpha_{6}+\alpha_{8}+\alpha_{9}+\alpha_{10}+\alpha_{11}, \beta=$ $\alpha_{1}+\alpha_{2}+\alpha_{4}+\alpha_{6}+\alpha_{7}+\alpha_{8}+\alpha_{9}+\alpha_{10}+\alpha_{11}$ and $\gamma=\alpha_{1}+\alpha_{2}+\alpha_{3}+\alpha_{4}+\alpha_{7}+\alpha_{9}+\alpha_{11}$. Finally we compute a preimage of $h$ as

$$
m^{\prime}=m_{\alpha} 0 m_{\beta} 000 m_{\gamma}
$$


where

$$
\begin{aligned}
& m_{\alpha}=\mu_{L}\left(m_{1}\right) \mu_{L}\left(m_{4}\right) \mu_{L}\left(m_{6}\right) \mu_{L}\left(m_{8}\right) \mu_{L}\left(m_{9}\right) \mu_{L}\left(m_{10}\right) \mu_{L}\left(m_{11}\right), \\
& m_{\beta}=\mu_{U}\left(m_{1}\right) \mu_{U}\left(m_{2}\right) \mu_{U}\left(m_{4}\right) \mu_{U}\left(m_{6}\right) \mu_{U}\left(m_{7}\right) \mu_{U}\left(m_{8}\right) \mu_{U}\left(m_{9}\right) \mu_{U}\left(m_{10}\right) \mu_{U}\left(m_{11}\right), \\
& m_{\gamma}=\mu_{L}\left(m_{1}\right) \mu_{L}\left(m_{2}\right) \mu_{L}\left(m_{3}\right) \mu_{L}\left(m_{4}\right) \mu_{L}\left(m_{7}\right) \mu_{L}\left(m_{9}\right) \mu_{L}\left(m_{11}\right) .
\end{aligned}
$$

\section{B Further Experimental Results on Our First Precomputing Algorithm}

Since it is unlikely that we can give a better theoretical analysis of our first precomputing algorithm, we provide additional experimental results in this section.

The results shown in Figure 1 were obtained with each "shortest" polynomial of degree $n$. Figure 2 shows similar results for randomly chosen polynomials. The algorithm always succeeds with a value $R$ satisfying $\frac{2^{R}}{R} \geq 6 n$. For reasonably large $n$, it always succeeds when $\frac{2^{R}}{R} \geq 4 n$. When $n$ increases the points get closer to the bound $\frac{2^{R}}{R} \geq 2 n$. Some of the points of Figure 1 and Figure 2 differ, but the differences are small and they only appear for small $n$.

\begin{tabular}{|c|c||c|c||c|c|}
\hline$n$ & $R$ & $n$ & $R$ & $n$ & $R$ \\
\hline 5 & 7 & 67 & 11 & 257 & 13 \\
7 & 7 & 73 & 11 & 277 & 13 \\
11 & 9 & 83 & 11 & 307 & 13 \\
13 & 9 & 97 & 12 & 331 & 14 \\
17 & 9 & 103 & 12 & 353 & 14 \\
19 & 9 & 109 & 12 & 379 & 14 \\
23 & 9 & 127 & 12 & 401 & 14 \\
29 & 10 & 137 & 12 & 449 & 14 \\
31 & 9 & 149 & 12 & 499 & 14 \\
37 & 10 & 167 & 12 & 607 & 15 \\
41 & 10 & 191 & 13 & 1021 & 15 \\
47 & 11 & 211 & 13 & 2039 & 16 \\
59 & 11 & 233 & 13 & & \\
\hline
\end{tabular}

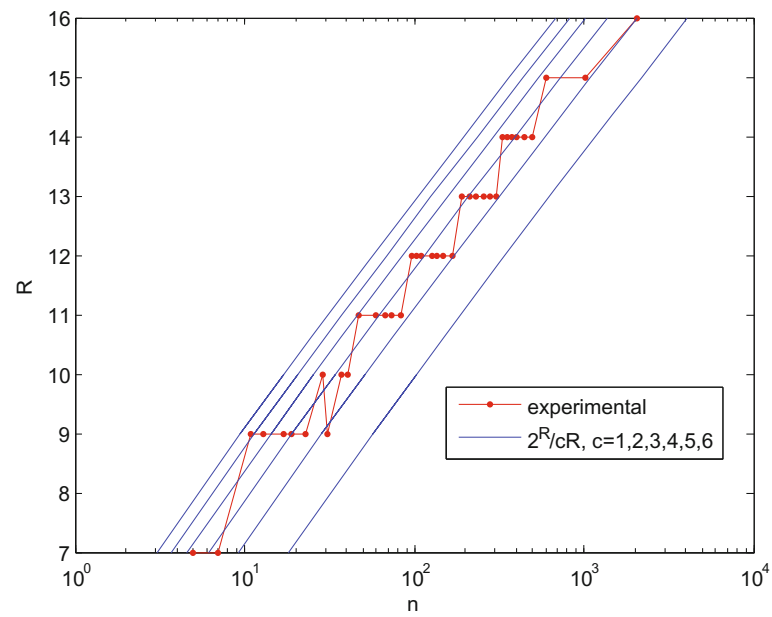

Fig. 2. Minimal value $R$ for different values $n$ and random polynomials. The points on the staircase-like curve are experimental results. The other curves are $n=\frac{2^{R}}{c R}$ for $c=1,2,3,4,5,6$.

To study these differences in more details, we generated twenty random polynomials of degrees $n=11, n=47$ and $n=127$. For each polynomial, we recorded the shortest value of $R$ for which the algorithm succeeded. (We started with short $R$ values and increased $R$ when $1000 \alpha$ values linearly dependent with the previous ones were found). The results are presented in Table 1 The minimal value $R$ for the success of the algorithm does not depend very much on the parameter $p$ but only on its degree. Moreover, this dependence seems to 
Table 1. Variability of the minimal $R$ needed for the algorithm when the polynomial $p$ is randomly chosen. For each degree $n$ and each $R$ value, the table indicates how many polynomials $p$ among the 20 polynomials generated required at least a randomness $R$.

\begin{tabular}{|r|r|r|r|r|r|}
\hline \multicolumn{2}{|c|}{$n=11$} & \multicolumn{2}{|c|}{$n=47$} & \multicolumn{2}{c|}{$n=127$} \\
\hline$R=7$ & $3 / 20$ & $R=10$ & $11 / 20$ & $R=12$ & $20 / 20$ \\
$R=8$ & $14 / 20$ & $R=11$ & $9 / 20$ & & \\
$R=9$ & $3 / 20$ & & & & \\
\hline
\end{tabular}

disappear for reasonably large degrees $n$. Therefore, the algorithm is likely to succeed for any $p$ as long as $\frac{2^{R}}{R} \geq 4 n$ (for reasonably large $n$ values).

Choosing $R$ close to the minimal value will improve the efficiency of the algorithm since it performs linear algebra on vectors of length $N+R$. On the other hand, if $R$ is chosen too close to the minimum, the algorithm will have to generate more polynomials $p^{\prime}$ before getting $n$ independent elements $\alpha_{i}$. In Table 2, it seems more efficient to choose $R=10$ instead of $R=8$ for the polynomial $p(X)=X^{11}+X^{2}+1$, and $R=12$ instead of $R=10$ for the polynomial $p(X)=X^{47}+X^{5}+1$. The reason appears clearly in the toy example of the previous section, presented in Table 1, in this example, all the dependencies obtained between the $\alpha$ values do actually come from the fact that the same polynomials are generated various times.

To conclude this section, we observe that in the experiments of Table 2, the Mesirov and Sweet's system had solutions respectively $49,21 \%$ and $51,36 \%$ of times for the polynomials $p(X)=X^{11}+X^{2}+1$ and $p(X)=X^{47}+X^{5}+1$. This confirms the analysis of Section 5.1 .

Table 2. Number of iterations needed to obtain a basis of $K$, for various $R$ and the polynomials $p(X)=X^{11}+X^{2}+1$ and $p(X)=X^{47}+X^{5}+1$. The first row gives the total number of polynomials generated, the second row gives the number of times the Mesirov and Sweet's system had no solution, and the last row gives the number of times a new $\alpha$ value was linearly dependent on the previous ones. For each value $R$, we performed the experiment three times.

\begin{tabular}{|c|c|c|c|c|c|c|c|c|c|c|c|c|c|c|c|c|}
\hline \multicolumn{17}{|c|}{$p(X)=X^{11}+X^{2}+1$} \\
\hline & $R=$ & & & $=$ & & & $\overline{=1}$ & & & $\overline{=}$ & & & $=2$ & & $R=5$ & \\
\hline \# pol. generated & \begin{tabular}{|l|l|}
48 & 36 \\
\end{tabular} & 25 & 41 & 61 & & 28 & 24 & $\overline{38}$ & 35 & 23 & 19 & 27 & 26 & 17 & & 22 \\
\hline sol. MS & $21 \mid 9$ & 6 & $28 \mid$ & 44 & & 15 & 8 & 22 & 22 & 11 & 8 & 14 & 11 & 6 & $11 \mid 13$ & 10 \\
\hline \# dependencies & $16 \quad 16$ & 8 & 2 & 6 & & 2 & 5 & 5 & 2 & 1 & 0 & 2 & 4 & 0 & \begin{tabular}{|l|l}
3 & 4 \\
\end{tabular} & 1 \\
\hline
\end{tabular}

\begin{tabular}{|c|c|c|c|c|c|c|c|c|c|c|c|c|c|c|c|c|c|}
\hline \multicolumn{18}{|c|}{$p(X)=X^{47}+X^{5}+1$} \\
\hline & \multicolumn{3}{|c|}{$R=10$} & \multicolumn{3}{|c|}{$R=11$} & \multicolumn{3}{|c|}{$R=12$} & \multicolumn{3}{|c|}{$R=15$} & \multicolumn{2}{|c|}{$R=20$} & \multicolumn{3}{|c|}{$R=50$} \\
\hline \# pol. g & 211 & 293 & 209 & 119 & 110 & 110 & 100 & 131 & 101 & 94 & 107 & 93 & \begin{tabular}{|l|l|}
89 & 98 \\
\end{tabular} & 87 & 104 & 105 & 111 \\
\hline$\# \mathrm{r}$ & 103 & 150 & 103 & 47 & 46 & 46 & 44 & 73 & 53 & 44 & 55 & 44 & 4050 & 38 & 54 & 54 & 61 \\
\hline \# dependencies & 61 & 96 & 59 & 25 & 17 & 17 & 9 & 11 & 1 & 3 & 5 & 2 & $2 \mid \begin{array}{ll}2 & 1\end{array}$ & 2 & 3 & 4 & 3 \\
\hline
\end{tabular}

